\title{
Guaranteed performance in reaching mode of sliding mode controlled systems
}

\author{
G K SINGH ${ }^{1}$ and K E HOLÉ 2 \\ ${ }^{1}$ Flight Mechanics and Control Division, National Aerospace Laboratories, \\ Bangalore 560 017, India \\ ${ }^{2}$ Department of Electrical Engineering, Indian Institute of Technology, \\ Kanpur 208 016, India \\ e-mail: gksingh@css.cmmacs.ernet.in
}

MS received 26 July 2002; revised 1 July 2003

\begin{abstract}
Conventionally, the parameters of a sliding mode controller (SMC) are selected so as to reduce the time spent in the reaching mode. Although, an upper bound on the time to reach (reaching time) the sliding surface is easily derived, performance guarantee in the state/error space needs more consideration. This paper addresses the design of constant plus proportional rate reaching law-based SMC for second-order nonlinear systems. It is shown that this controller imposes a bounding second-order error-dynamics, and thus guarantees robust performance during the reaching phase. The choice of the controller parameters based on the time to reach a desirable level of output tracking error (OTE), rather than on the reaching time is proposed. Using the Lyapunov theory, it is shown that parameter selections, based on the reaching time criterion, may need substantially larger time to achieve the OTE. Simulation results are presented for a nonlinear spring-massdamper system. It is seen that parameter selections based on the proposed OTE criterion, result in substantially quicker tracking, while using similar levels of control effort.
\end{abstract}

Keywords. Reaching mode; sliding mode controlled systems; output tracking error; Lyapunov theory.

\section{Introduction}

Sliding mode control (SMC) originated in the Soviet literature (Utkin 1978). Some survey/review on this topic can be found in the references, Utkin (1977), DeCarlo et al (1988), Hung et al (1993), and more recently by Young et al (1997). The SMC design method involves two steps, (i) the selection of a stable hyperplane in the state/error space on which motion should be restricted, called the sliding surface (SS), and (ii) the synthesis of a control law which makes the selected surface attractive. In this method a trajectory, starting from a nonzero initial condition, evolves in two phases: 
- A reaching phase, in which it reaches the SS, and

- A sliding phase, in which the trajectory on reaching the SS, remains there for all further times and thus evolves according to the dynamics specified by the SS.

Invariance to matched external disturbances and uncertainties is guaranteed (Drazenovic 1969) during the sliding phase. The system evolution in the reaching phase depends on the system uncertainties and the selected reaching laws. A smaller reaching time would imply that sliding begins earlier and this has been conventionally used to select the SMC parameters.

Many mechanical systems are modeled as second-order systems e.g. as in the trajectory following robots, or the angular position control in aircrafts as nose pointing or pitch hold autopilots. Other examples include the classical inverted pendulum, or the antenna pointing of satellites among others. The SMC method has been used for many such applications (Gao \& Hang 1993; Hung et al 1993; Young et al 1999). Thus it is of particular interest to study the effect of the SMC parameters for this class of systems. Gao \& Hung (1993) propose a reaching law method which specifies the error dynamics in the reaching phase. They suggest three structures - the constant rate reaching, constant plus proportional rate $(C+P R)$, and the power rate reaching. The time taken to reach the SS is also evaluated. By appropriately selecting parameters the dynamic quality of the SMC system can be controlled. Choi et al (1994) suggest the use of a stepwise time-varying SS, in which the SS is shifted and/or rotated as a function of time. They applied this method to a nonlinear spring mass damper system and reported a substantial decrease in time to achieve zero tracking error. However, the resulting controller is cumbersome to implement because the parameters of the SS need to be calculated at each time instant. Bartoszewicz (1995) proposed a continuously time-varying SS that gives still faster tracking. The choice of the time varying SS is extended by Bartoszewicz (1995) so as to minimize the integral of the absolute error. However, the parameter selection is dependent on the initial error conditions. A similar approach is reported by Yilmaz \& Hurmuzlu (2000) to eliminate the reaching phase from SMC. Mantz et al (2001) used trajectory planning strategy for evolution during the reaching phase for differentially flat systems.

This paper studies the design of constant plus proportional rate reaching law $(\mathrm{C}+\mathrm{PR}-\mathrm{RL})$ for second-order systems. Using Lyapunov theory, it is established in $\S 3$. that $C+P R-R L$ imposes a bounding second-order dynamics on the error variables and thus guarantees a robust performance in the reaching phase also. It is shown that the selection of controller parameters based on minimizing the reaching time may not result in smaller times to reach a desired level of output tracking error (OTE). A new method of selection of the control law parameters based on the criterion of time to a specified OTE is proposed. To illustrate the method, it is applied to a nonlinear spring mass problem (Choi et al 1994). Simulation results show that the proposed method of selection of controller parameters results in considerably faster tracking performances, for similar peak levels of control effort.

\section{The reaching law-based SMC design method}

Consider a single input, second-order input-affine nonlinear system of the form

$$
\ddot{x}=f(x, \dot{x})+g(x, \dot{x}) u+d_{f},
$$

where $u$ is the control input, and $d_{f}$ represents the uncertainties and external disturbances which are assumed to be bounded with $\left|d_{f}\right|<D$. Since the uncertainties and/or disturbances which are in the range space of the input are called matched, it is obvious that any uncertainty 
entering the scalar system as given above is matched. The arguments of $f$ and $g$ are dropped from now on for brevity. The uncertainty in the input distribution function $g$ is expressed as $g=\hat{g}(1+\tilde{g})$, where $\hat{g}$ represents its nominal value, and the uncertainty $\tilde{g}$ is bounded as $|\tilde{g}|<G$, with $G<1 \cdot 0$.

Defining the state variables as $x_{1}=x$ and $x_{2}=\dot{x},(1)$ can be written in the state space form as

$$
\left(\begin{array}{c}
\dot{x}_{1} \\
\dot{x}_{2}
\end{array}\right)=\left(\begin{array}{c}
x_{2} \\
f+g u+d_{f}
\end{array}\right)
$$

Let the trajectory to be followed (desired trajectory) be given as $x_{1 d}$ and $x_{2 d}=\dot{x}_{1 d}$. Then the error between the actual and the desired trajectories can be expressed as $e=x_{1}-x_{1 d}$ and $\dot{e}=x_{2}-x_{2 d}$. In vector form,

$$
y=\left(\begin{array}{c}
e \\
\dot{e}
\end{array}\right)=\left(\begin{array}{c}
x_{1}-x_{1 d} \\
x_{2}-x_{2 d}
\end{array}\right) .
$$

Taking the time derivative of the above equation and using results of (2) in the following error-dynamics,

$$
\dot{y}=\left(\begin{array}{c}
\dot{e} \\
\ddot{e}
\end{array}\right)=\left(\begin{array}{c}
\dot{e} \\
f+g u+d_{f}-\ddot{x}_{1 d}
\end{array}\right) .
$$

The switching function $s$ for second-order systems is conventionally defined as a combination of the error variables (defined in (3)) (Slotine \& Li 1991; Gao \& Hung 1993) as

$$
s=\dot{e}+\alpha e,
$$

where, $\alpha>0$ sets the dynamics in the sliding phase $(s=0)$. The SS selected above should be made attractive so that trajectories starting from a non-zero value of $s$ approach the SS, and then stay on $s=0$ for all future time instants. For this, using $\ddot{e}$ from (4), the time derivative $\dot{s}$ is obtained from (5) as

$$
\begin{aligned}
\dot{s} & =\ddot{e}+\alpha \dot{e}, \text { or } \\
& =f+g u+d_{f}-\ddot{x}_{1 d}+\alpha \dot{e}, \\
& =f+\hat{g}(1+\tilde{g}) u+d_{f}-\ddot{x}_{1 d}+\alpha \dot{e} .
\end{aligned}
$$

The input $u$ remains to be chosen so as to make $s$ go to zero for sliding. The normal procedure for its selection (Slotine \& Li 1991) is to express the input $u$ as a sum of two terms. The first term is chosen, using the nominal plant parameters, so as to make $\dot{s}=0$, when $s=0$. This is called the equivalent control and is seen to be $\hat{g}^{-1}\left(\ddot{x}_{1 d}-f-\alpha \dot{e}\right)$. The second term is then chosen to tackle the uncertainties in the system and introduce a reaching law. The constant plus proportional rate reaching law (Gao \& Hung 1993) is imposed by selecting the second term as $\hat{g}^{-1}(-\epsilon s-K \operatorname{sgn}[s])$. The complete $u$ thus becomes

$$
u=\hat{g}^{-1}\left(\ddot{x}_{1 d}-f-\alpha \dot{e}-\epsilon s-K \operatorname{sgn}[s]\right),
$$

where, $\epsilon, K>0$ are positive real numbers to be selected, and sgn[·] is the signum function.

Note that the $u$ in the above equation may be simplified by using the definition of $s$ in (5) and defining $a$ and $b$ as

$$
\begin{aligned}
& a=\alpha \epsilon, \text { and } \\
& b=\alpha+\epsilon,
\end{aligned}
$$


both of which are obviously positive real numbers. Thus $u$ becomes

$$
u=\hat{g}^{-1}\left(\ddot{x}_{1 d}-f-a e-b \dot{e}-K \operatorname{sgn}[s]\right) .
$$

The positive scalar $K$ should be chosen such that the time evolution of $s$ satisfies the $\mathrm{C}+$ PR - RL as,

$$
\dot{s}=-\epsilon s-\eta \operatorname{sgn}[s],
$$

where, $\eta$ is a positive real number to be selected later. Thus, $K$ may be selected (Slotine $\&$ Li 1991) as

$$
K=\frac{G|R|+D+\eta}{1-G} .
$$

where,

$$
R=\ddot{x}_{1 d}-f-a e-b \dot{e} .
$$

With $K$ as selected above, the switching function $(s)$ evolves as,

$$
\dot{s}=-\epsilon s-\eta^{\prime} \operatorname{sgn}[s],
$$

where $\eta^{\prime} \geq \eta$. Note that $\eta^{\prime}$ as defined above can be shown to be bounded with a maximum possible value of $\eta_{M}=\eta+2 K$.

Furthermore, the reaching time, i.e. the time taken to arrive at $s=0$ starting from $s_{0}$ for the $s$ dynamics (10) is bounded (Gao \& Hung 1993) as

$$
t_{\text {reach }} \leq \frac{1}{\epsilon} \ln \left(\frac{\epsilon\left|s_{0}\right|+\eta}{\eta}\right),
$$

This has been conventionaly used to select the SMC parameters, since it gives an upper bound on the time the system (1) spends in the reaching phase. Note that $K$ specifies the magnitude of the discontinuity in the control law in (9). The value of $K$ increases with $\eta$. For a given value of $\epsilon$, a higher value of $\eta$ leads to smaller reaching times as seen from the above equation, it also results in more chattering (Gao \& Hung 1993). There have been efforts to reduce the chattering problem by either applying a boundary layer technique (Slotine \& Li 1991) or by adaptively estimating the upper bounds on the uncertainties (Wheeler et al 1998). This is however not in the scope of the current study. The effect of the controller parameters, namely the positive scalars $\alpha, \epsilon$ and $\eta$ are studied in the following section.

\section{Robustness in reaching phase}

It is a commonly held belief that the conventional reaching laws impose conditions on the time evolution of the switching function, rather than on the state/error dynamics (Mantz et al 2001). A Lyapunov approach is used in this section to show that robust performance can be guaranteed during the reaching mode also.

The dynamics in the error space are given in (4). Note that, $\ddot{e}=\dot{s}-\alpha \dot{e}$ from (6). Substituting $\dot{s}$ from (13),

$$
\ddot{e}=-a e-b \dot{e}-\eta^{\prime} \operatorname{sgn}[s],
$$


with $a$ and $b$ as defined in (8). Thus, the error dynamics $y$, may be written as

$$
\dot{y}=A y+\bar{q},
$$

where,

$$
A=\left[\begin{array}{cc}
0 & 1 \\
-a & -b
\end{array}\right]
$$

and,

$$
\bar{q}=\left(\begin{array}{c}
0 \\
-\eta^{\prime} \operatorname{sgn}[s]
\end{array}\right) .
$$

With $a$ and $b$ defined as in (8), the $A$ matrix above is seen to have its eigenvalues at $-\alpha$ and $-\epsilon$. These poles govern the response of the linear homogeneous part (i.e. the $A$-dynamics).

A Lyapunov approach is adopted to evaluate the effect of $\alpha$ and $\epsilon$ parameters on the tracking performance. A candidate Lyapunov function $V$ for the $y$ dynamics as specified in (16) is selected as

$$
V(y)=y^{\mathrm{T}} P y,
$$

where $P$ is a positive definite symmetric matrix and is taken as

$$
P=\left[\begin{array}{cc}
\alpha^{2}+\beta & \alpha \\
\alpha & 1
\end{array}\right],
$$

and $\beta>0$ guarantees the positive definiteness of the $P$ matrix. The time derivative of $V$ may be obtained as

$$
\dot{V}=y^{\mathrm{T}}\left[A^{\mathrm{T}} P+P A\right] y+2 y^{\mathrm{T}} P \bar{q} .
$$

Since $A$ in (17) is a Hurwitz matrix, it satisfies the Lyapunov equation

$$
A^{\mathrm{T}} P+P A=-Q,
$$

where $Q$ can be derived from (22) using $A$ and $P$ as given in (17) and (20) as

$$
Q=\left[\begin{array}{cc}
2 \alpha^{2} \epsilon & 2 \alpha \epsilon-\beta \\
2 \alpha \epsilon-\beta & 2 \epsilon
\end{array}\right] .
$$

Note that positive definiteness of $Q$ is guaranteed if

$$
\beta<4 \alpha \epsilon \text {. }
$$

From $\bar{q}$ and $P$ as defined in (18) and (20) respectively, the $y^{\mathrm{T}} P \bar{q}$ is seen to be equal to $-s \eta^{\prime} \operatorname{sgn}[s]$. Using this and $Q$ as obtained above in (23), $\dot{V}$ in (21) may be simplified to

$$
\begin{aligned}
\dot{V} & =-y^{\mathrm{T}} Q y-2 s \eta^{\prime} \operatorname{sgn}[s], \text { or } \\
& =-y^{\mathrm{T}} Q y-2 \eta^{\prime}|s|,
\end{aligned}
$$

which is seen to be always negative definite. Thus, the stability of $e$ dynamics in (16) or equivalently in (15) is guaranteed. To study the time decay performance of $V$ in the above equation, the following result is required. 
Claim 1. For the second-order error dynamics as given in (15), a Lyapunov function may be selected as in (19). For the $Q$ matrix in (23), as obtained from the Lyapunov equation (22), there exists a positive real number $\delta>0$ such that

$$
Q=\delta P+H,
$$

where $H$ is at least a positive semi-definite matrix.

Proof. It can be easily shown by a constructive proof that for the system under consideration, there is always a range of values that $\delta$ can take. The detailed proof is presented by Singh (1999). In particular, it is established that permissible range of values of $\delta$ lies in an open set and is given by $\delta \in(0, \min [2 \alpha, 2 \epsilon])$, where $\min [\cdot, \cdot]$ gives the minimum of its arguments.

Using the decomposition of $Q$ as in (26), the $\dot{V}$ in (25) may be rewritten as

$$
\dot{V}=-\delta V-y^{\mathrm{T}} H y-2 \eta^{\prime}|s| \text {. }
$$

It follows that the $V$ decay response of the above system will be better than the response of the system described by

$$
\dot{V}=-\delta V \text {. }
$$

It is interesting to find out the $A_{b}$ matrix with the dynamics specified as

$$
\dot{y}=A_{b} y,
$$

that satisfies (28). The $A_{b}$ should thus satisfy the Lyapunov equation (22) with the $P$ as selected in (20), i.e. $A_{b}^{\mathrm{T}} P+P A_{b}=-\delta P$, for some feasible choice of $\delta>0$ as in (28). For this, the general form of the $A_{b}$ matrix is taken as

$$
A_{b}=\left[\begin{array}{cc}
0 & c^{\prime} \\
-a^{\prime} & -b^{\prime}
\end{array}\right],
$$

where, $a^{\prime}, b^{\prime}, c^{\prime}$ are to be determined. The Lyapunov equation for $A_{b}$ matrix as given above is solved for these unknowns for a general value of $\delta$. These may be easily obtained by the solution of a set of simultaneous linear equations in $a^{\prime}, b^{\prime}, c^{\prime}$ as

$$
\begin{aligned}
& a^{\prime}=\delta\left(\alpha^{2}+\beta\right) / 2 \alpha \\
& b^{\prime}=\delta \\
& c^{\prime}=\delta / 2 \alpha .
\end{aligned}
$$

It is known from claim 1 that $\delta \in(0, \min [2 \alpha, 2 \epsilon])$. If $\alpha<\epsilon$, the $A_{b}$ matrix is obtained by using $\delta=2 \alpha$ as

$$
A_{b}=\left[\begin{array}{cc}
0 & 1 \\
-\left(\alpha^{2}+\beta\right) & -2 \alpha
\end{array}\right],
$$

and is seen to have both of its eigenvalues at $-\alpha$, for $\beta \rightarrow 0$. Alternately, if $\alpha>\epsilon$, then the $A_{b}$ matrix is obtained by using $\delta=2 \epsilon$, as

$$
A_{b}=\left[\begin{array}{cc}
0 & \epsilon / \alpha \\
-(\epsilon \alpha+\epsilon \beta / \alpha) & -2 \epsilon
\end{array}\right],
$$


and for $\beta \rightarrow 0$, both the eigenvalues of the above $A_{b}$ matrix are easily seen to lie at $-\epsilon$. It follows that the decay of the Lyapunov function (19) for the dynamics in (16) with the $A$ matrix in (17) having its eigenvalues as $-\alpha$ and $-\epsilon$, is guaranteed to be better than the decay response in (28), which is achievable by a $A_{b}$ matrix as given in (32) or (33) which has both of its eigenvalues at $-\min [\alpha, \epsilon]$. This implies that the error dynamics in (16) in the reaching phase is bounded by the response of the second-order $A_{b}$ dynamics in (29) and a robust performance in the presence of the matched uncertainties and disturbances is achievable in the reaching mode even before the onset of sliding. Estimates of the guaranteed times to reach the OTE may be obtained using the $A_{b}$ dynamics. It is obvious that shorter times to the OTE are expected as both $\alpha$ and $\epsilon$ are increased. However, the control law in (9) will also require larger control magnitudes as $a$ and $b$ will also increase as can be seen from (8). Next the effect of the relative values of these parameters is considered.

\subsection{Selection of $\alpha$ and $\epsilon$}

Let the eigenvalues of the A matrix in (17) be selected as $-p$ and $-q$, where $p$ and $q$ are positive real numbers satisfying $p>q$. This could be achieved in the following two ways:

- Case $C P: \alpha=p$ and $\epsilon=q$. This would result in the SS being $\dot{e}+p e$, say $S_{p}$.

- Case $C Q: \alpha=q$ and $\epsilon=p$, with which the SS becomes $\dot{e}+q e$, say $S_{q}$.

It follows from (8) that $a=p q$ and $b=p+q$ remain the same in both the cases resulting in the same $A$ matrix in (17). With $a$ and $b$ being the same, $R$ in (12) and thus $K$ in (11) are expected to have comparable magnitudes for both the cases. Thus the peak values of the control effort $u$ in (9) should also be nearly equal. The performance obtained in the two cases is compared next. For this, a common Lyapunov function is first constructed as follows.

Claim 2. Let $P$ and $Q$ be symmetric positive definite matrices as specified in (20) and (23) respectively, for the case $C P$, i.e. $\alpha=p$ and $\epsilon=q$. Then, there exists a sufficiently large positive scalar $M$ such that

$$
V(y)=y^{\mathrm{T}} P y+M\left|S_{p}\right|+M\left|S_{q}\right|
$$

where, $y=(e, \dot{e})^{\mathrm{T}}$, is a valid common Lyapunov function for cases $C P$ and $C Q$.

Proof. Note that this candidate function is not differentiable on the planes $S_{p}=0$ and $S_{q}=0$. However, it is possible to use the generalized Lyapunov theorem, which allows nondifferentiability of the Lyapunov function in a set of measure zero (Singh 1989; Chiacchiarini et al 1995). For $V$ to be a valid common Lyapunov function, at least negative semi-definiteness (n.s.d.) of its time derivative must be established for the two cases. The detailed proof is presented by Singh (1999).

Let $\dot{V}_{i}, i=p, q$ represent the time derivative $\dot{V}$ of the Lyapunov function in (34) for the cases denoted by the subscript ' $i$ '. Further, for the case $C Q$, it may be shown that there exists a $0<\delta_{q}<q\left(<p\right.$, by selection), using which $\dot{V}_{q}$ may be written as

$$
\dot{V}_{q} \leq-y^{\mathrm{T}} Q y-\delta_{q} M\left[\left|S_{q}\right|+\left|S_{p}\right|\right]-\left(p-\delta_{q}\right) M\left|S_{q}\right| .
$$

From claim 1, it is known that the $Q$ matrix may be decomposed as given in (26) as $Q=$ $\delta_{q} P+H_{q}$, where $H_{q}$ is a positive definite matrix, since $\delta_{q}<q<\min [2 q, 2 p$ ]. Using this decomposition, the $\dot{V}_{q}$ above may be expressed as

$$
\dot{V}_{q} \leq-\delta_{q} V_{q}-\left(p-\delta_{q}\right) M\left|S_{q}\right|,
$$


using the definition of $V$ in (34) where the subscript ' $q$ ' represents the case $C Q$. As $p>q>$ $\delta_{q}$, the $\left(p-\delta_{q}\right) M\left|S_{q}\right|$ term in the above equation is always non-negative. Thus, the $V_{q}$ decay response of this system will be better than the exponential decay response offered by

$$
\dot{V}_{q}=-\delta_{q} V_{q}, \text { where } \delta_{q} \in(0, q) \text {. }
$$

Note that $\delta_{q}$ can assume a maximum value of $\bar{\delta}_{q}=q$ in the limit.

For the case $C P, \dot{V}_{p}$ may be obtained as

$$
\dot{V}_{p} \leq-y^{T} Q y-M\left[q\left|S_{p}\right|+p\left|S_{q}\right|\right]-2 \eta^{\prime}\left|S_{p}\right| .
$$

The $Q$ matrix in this case is decomposable as $\delta_{p} P+H_{p}$, where $0<\delta_{p}<2 q=\min [2 q, 2 p$ ] has to be found out. Following the steps as for the case $C Q$, it may be shown that the $\delta_{p}$ may be selected to be smaller than $\min [p, 2 q]$. It thus follows that the $V_{p}$ decay response of the above system is better than the response of the system given by

$$
\dot{V}_{p}=-\delta_{p} V_{p}, \text { where } \delta_{p} \in(0, \min [p, 2 q]) .
$$

Thus the upper limiting value of $\delta_{p}$ is seen to be given by $\bar{\delta}_{p}=\min [p, 2 q]$. Thus it is obvious that $\bar{\delta}_{p}>\bar{\delta}_{q}$.

With the guaranteed decay responses of $V_{q}$ and $V_{p}$ being given as in (37) and (39) respectively, it is clear that case $C P$ guarantees a faster exponential decay of the Lyapunov function (34) as compared to case $C Q$. These guaranteed decay performances may be used to develop an estimate of the time to reach a specified level of OTE in terms of the Lyapunov function. Thus, given an initial value $V(0)$ at $t=0$, the time $t_{f}$ required for $V_{i}, \mathrm{i}=q, p$ dynamics, in (37) and (39) respectively, to decay to a final value of $V_{f}$ may be evaluated as

$$
t_{f}=\left(1 / \bar{\delta}_{i}\right) \ln \left[V(0) / V_{f}\right],
$$

where, $\mathrm{i}=q, p$, and $\bar{\delta}_{i}$ are as defined earlier and represent the maximum limiting value of $\delta_{i}$ for the $\delta_{q}$ and $\delta_{p}$ as given in (37) and (39) respectively. For the same $V(0) / V_{f}$, it is obvious that $t_{f}$ in the case $C P$ ill be smaller than in the case $C Q$, as $\bar{\delta}_{p}>\bar{\delta}_{q}$. The $t_{f}$ in (40) gives conservative estimates for the decay of the Lyapunov function. The actual times $t_{O T E}$ required, for the $V$ decay responses in (36) and (38) for the cases $C Q$ and $C P$ respectively, to reach the $V_{f}$ are expected to be smaller than the $t_{f}$, i.e. $t_{O T E} \leq t_{f}$. Thus the $\alpha$ and $\epsilon$ parameters should be selected as in the case $C P$ to have a smaller value of $t_{f}$, i.e. for a given choice of the eigenvalues of the $A$ matrix in (17), $\epsilon$ should be selected as the magnitude of the dominant pole, and $\alpha$ as the magnitude of the other, or simply $\alpha>\epsilon$. This is summarized as follows.

Lemma 1. The choice of $\alpha$ and $\epsilon$ parameters, for the constant plus proportional rate reaching SMC law as in (9), with a and b selected as in (8) and the sliding surface as in (5), such that

$$
\alpha>\epsilon \text {. }
$$

guarantees a faster decay of the Lyapunov function (34).

Note that the above lemma indicates that the choice of $\alpha$ and $\epsilon$ as in (41) only ensures that $t_{f}$ is smaller. It however does not guarantee that the $t_{O T E}$ for the case $C P$ is smaller than case $C Q$, 
although it is so expected and can actually be seen from the simulation results presented in the next section. There may however be parameter combinations where this would not hold.

A smaller value of $V$ implies a smaller tracking error in the sense of the norm (as defined by the Lyapunov function). Thus, since case $C P$ has a faster guaranteed decay performance, it is expected to take lesser time as compared to case $C Q$ to reach an acceptably small OTE. As discussed earlier, the control effort is expected to be almost the same for both the $C P$ and $C Q$ cases. Note that lower reaching times are obtained for the case of $\epsilon>\alpha$ (case $C Q$ ) as can be seen from the results in the next section. However, the above theoretical developments show that shorter times to a desired OTE are expected for the cases where $\alpha>\epsilon$ (case $C P$ ). The estimates of the time to the OTE using (40) showing the effect of the relative choices of $\alpha$ and $\epsilon$ as suggested in lemma 1 are verified by applying the design method to a nonlinear spring mass damper system in the next section.

\section{Simulation results}

For the illustration of the proposed SMC design method, the results presented in the previous section have been used to design controllers for two practical problems. The numerical results of these examples ascertain the validity of lemma 1.

\subsection{Nonlinear spring mass damper system}

The first example concerns a nonlinear mass-spring-damper system from Choi et al (1994). The governing differential equation is

$$
m \ddot{x}+v(\dot{x}, t)+\mu(x, t)=u(t)+d(t),
$$

where, $x, \dot{x}$ represent the position and the velocity of the mass $m, u($.$) represents the applied$ input force, and $d($.$) the disturbance signal. v(.,$.$) and \mu(.,$.$) represent the damping and$ spring force term respectively. The system parameters and functions are given as $m=1$, $f=f_{1}+f_{2}+f_{3}+f_{4}$, which are given as $f_{1}=\mu_{0} x, f_{2}=-\mu_{1} x^{3}, f_{3}=-v_{0} \dot{x}$, and $f_{4}=-v_{1} \dot{x}|\dot{x}|$ with $\mu_{0}=0.5, \mu_{1}=0.5, v_{0}=0.3$, and $v_{1}=0 \cdot 3$. The system also has uncertainties expressed as $a_{1} f_{1}+a_{2} f_{2}+a_{3} f_{3}+a_{4} f_{4}$ with $a_{1}=-.05+0.25 \sin (5 \pi t)$, $a_{2}=-.05+0.25 \sin (5 \pi t), a_{3}=-.05+0.15 \sin (7 \pi t)$, and $a_{4}=-.05+0.15 \sin (7 \pi t)$ and disturbance $d=0.05+0.25 \cos (3 \pi t)$. The signal to be tracked is given by $x_{1 d}=$ $-0.5 \cos (\pi t / 5)$. The above system in (42) may be expressed in standard form as in (1). The initial conditions are taken as 0.5 and 0 for the two states respectively.

Simulation was done using the fourth order Runge-Kutta method with a time step of $2.5 \mathrm{~ms}$. The remaining parameters were selected as $\beta=1$ and $\eta=1$. Several combinations of $\alpha$ and $\epsilon$ were used.

- Case 1: $\alpha=2$ and $\epsilon=6$

- Case 2: $\alpha=6$ and $\epsilon=2$

- Case 3: $\alpha=3$ and $\epsilon=4$

- Case 4: $\alpha=4$ and $\epsilon=3$

- Case 5: $\alpha=4$ and $\epsilon=5$

- Case 6: $\alpha=5$ and $\epsilon=4$

The time to OTE (defined as the time after which the error is less than $1.0 \times 10^{-3}$ ) obtained for the bounding $A_{b}$ dynamics in (29), the actual tracking times, the reaching times calculated 
Table 1. Comparison of time for OTE and reaching: example 1.

\begin{tabular}{|c|c|c|c|c|c|c|}
\hline \multirow[b]{2}{*}{$\begin{array}{l}\text { Case } \\
\text { no. }\end{array}$} & \multirow[b]{2}{*}{$\alpha$} & \multirow[b]{2}{*}{$\epsilon$} & \multicolumn{2}{|c|}{ Time to OTE $\left(<1 \times 10^{-3}\right)$ in $(\mathrm{s})$} & \multirow[b]{2}{*}{$\begin{array}{l}\text { Reaching time (s) } \\
\text { (14) }\end{array}$} & \multirow[b]{2}{*}{$\begin{array}{c}t_{f}(\mathrm{~s})(40) \\
\quad \text { for } \\
V_{0} / V_{f}=10^{4}\end{array}$} \\
\hline & & & $\begin{array}{l}\text { Bound obtained } \\
\text { from } A_{b} \text { dynamics (29) }\end{array}$ & Actual & & \\
\hline 1 & 2 & 6 & $4 \cdot 24$ & $3 \cdot 30$ & $0 \cdot 183$ & 4.605 \\
\hline 2 & 6 & 2 & $4 \cdot 24$ & $2 \cdot 00$ & 1.472 & $2 \cdot 302$ \\
\hline 3 & 3 & 4 & $2 \cdot 83$ & $2 \cdot 35$ & 0.426 & 3.070 \\
\hline 4 & 4 & 3 & 2.83 & $2 \cdot 00$ & 0.732 & $2 \cdot 302$ \\
\hline 5 & 4 & 5 & $2 \cdot 13$ & 1.80 & 0.439 & $2 \cdot 302$ \\
\hline 6 & 5 & 4 & $2 \cdot 13$ & 1.65 & 0.650 & 1.842 \\
\hline
\end{tabular}

from (14) and the $t_{f}$ for $V_{0} / V_{f}=1.0 \times 10^{4}$ as evaluated from (40) for all the cases are presented in table 1 . It can be seen from the table that the actual time to achieve the OTE is appreciably smaller for case 2 as compared to case 1 . Table 1 also shows that similar results are obtained for cases 4 and 6 . In all these cases $\alpha>\epsilon$, thus verifying lemma 1 . It is noticed from table 1 that, for all of these cases, the reaching time is greater than the corresponding cases 1, 3 and 5. Although cases 1, 3 and 5 have smaller reaching times, they require about $65 \%, 17.5 \%$, and $9.1 \%$ more time to OTE than the time required by cases 2,4 , and 6 respectively. Thus, from the table it is clearly evident that parameter selections giving lower reaching times actually take longer times to reach the specified OTE. Moreover, the time bounds $t_{f}$ calculated from (40) are seen to be better than those obtained from the $A_{b}$ dynamics (29) in the sense of being able to bring out the effect of the relative values of $\alpha$ and $\epsilon$.

Choi et al (1994) reported a time of $2.58 \mathrm{~s}$ to achieve a zero tracking error (OTE). It can be observed from table 1 that in almost all the cases under consideration, shorter tracking time is achieved by this approach. In fact the best tracking time achieved is $1.65 \mathrm{~s}$ for case 6 . In all of the cases the actual time to reach OTE is smaller than the bound given by the $A_{b}$ dynamics and $t_{f}$. As qualitatively similar responses are obtained, the responses obtained for the parameter combination in cases 1 and 2 are presented in figures 1,2, and 3 respectively. The responses are indexed by the case numbers in these figures. Note that case 1 corresponds

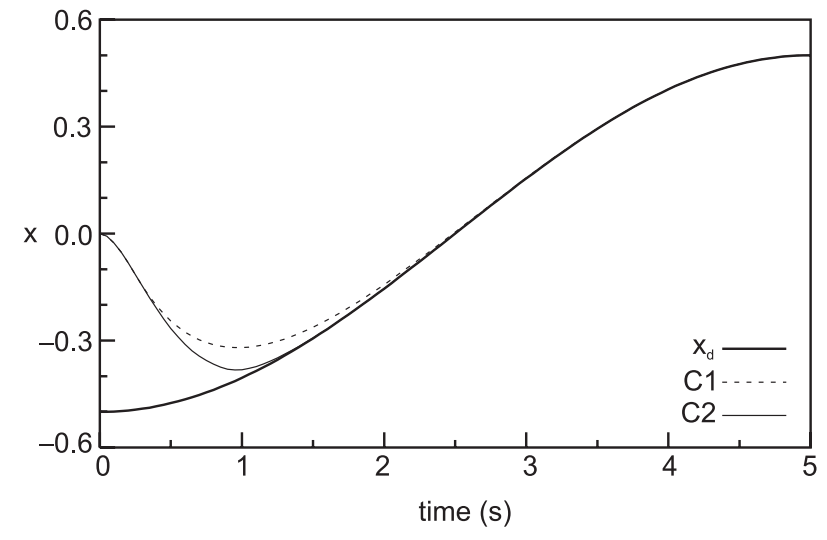

Figure 1. Tracking responses for case $1(\mathrm{C} 1)$ and case $2(\mathrm{C} 2)$. 


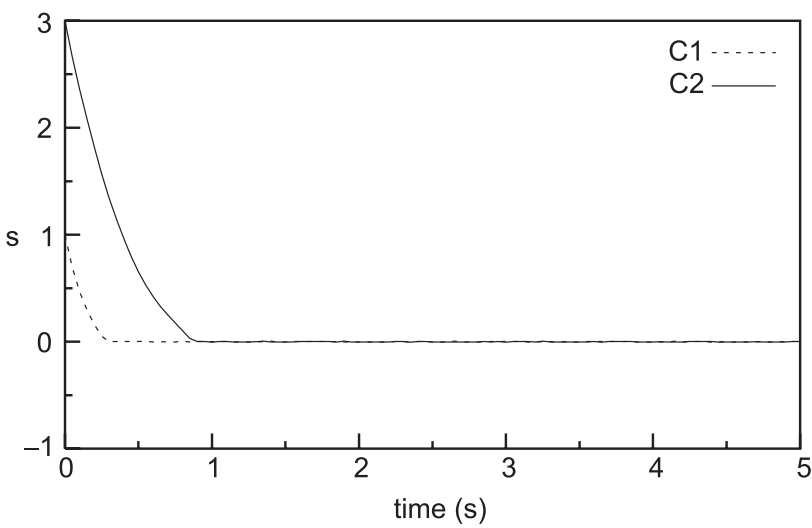

Figure 2. The $s$ responses for cases 1 and 2.

to case $C Q$, and case 2 to case $C P$. Figure 1 shows the tracking response obtained in the two cases. It is evident from the figure that the $\mathrm{C} 2$ (satisfying lemma 1) trajectory approaches the OTE in appreciably lower time as compared to the $\mathrm{C} 1$ trajectory. Figure 2 shows the $s$ response in the two cases. The $\mathrm{C} 1$ response has a lower reaching time (see also table 1) as compared to $\mathrm{C} 2$. Figure 3 shows the error decay with time for both the cases and also for the solution of the $A_{b}$ dynamics in (29), labeled as $e_{A}$. Although, only a better decay of the Lyapunov function in (19) is guaranteed for the control law in (9), the figure shows that the decay of $e$ obtained in the two cases are better than the solution of the $A_{b}$ dynamics in (29).

The required control effort in the two cases are shown in figure 4. As discussed in the previous section, since $a=12$ and $b=8$ from (8) are the same for the cases C1 and C2, the control efforts in these cases should be of about the same magnitude. This can indeed be seen from figure 4 , which verifies that that the proposed method uses about the same control effort to achieve the same tracking accuracy much faster. However as the reaching time in $\mathrm{C} 1$ is smaller, the input in case $\mathrm{C} 1$ begins chattering earlier as can be seen from the figure. Thus the selection of the parameters can be easily done drawing on the experience of the initial condition responses of linear second-order systems. If the $\alpha$ and $\epsilon$ are chosen so as to satisfy lemma 1, better results are expected.

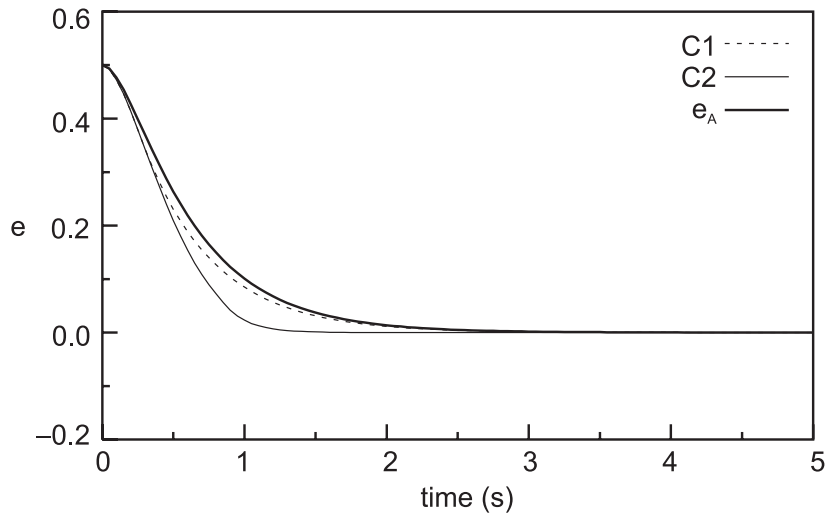

Figure 3. The error dynamics for cases 1 and 2 and that given by the $A$-dynamics $\left(e_{A}\right)$. 


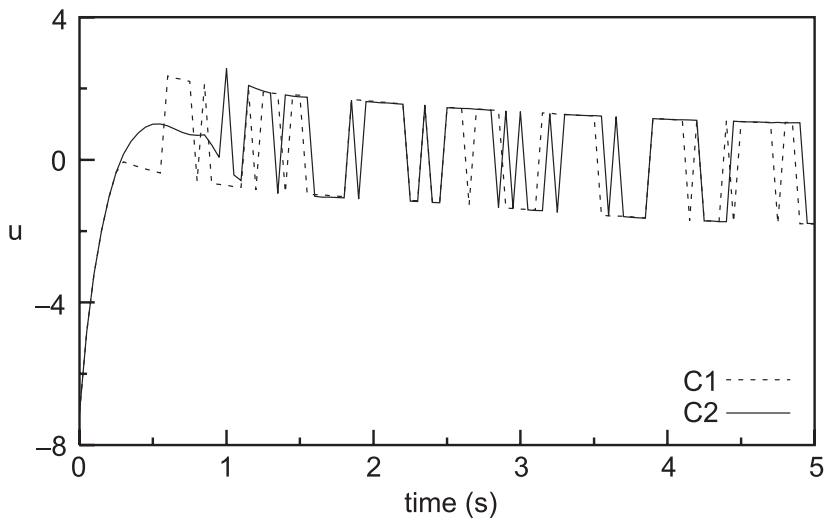

Figure 4. The control effort $u$ required for cases 1 and 2 .

\section{Conclusions}

In this paper the design of the constant plus proportional rate reaching SMC for second-order nonlinear systems has been studied. Investigation of the effect of the choice of the controller parameters has been done. Using a Lyapunov approach it has been shown that the design method imposes a bounding second-order dynamics on the error variables, and thus a robust performance in the reaching phase is guaranteed. A new method of selection of the SMC parameters which results in smaller times to reach a given level of OTE, while using almost similar levels of maximum control effort, is proposed. It is seen that for the class of systems under study, a small reaching time does not guarantee a fast tracking response. The method of parameter selection should thus be based on the time to OTE, rather than on the criterion of the reaching time. The design technique has been applied to a nonlinear spring-mass-damper system under matched uncertainties and exogenous disturbances. From the simulations done it is seen that the control laws based on the lower reaching times require approximately 10 $60 \%$ more time as compared to the proposed control laws to reach the same level of OTE, while using the same levels of control magnitudes. The method developed for a single input system has been extended to a two-input two-link robotic arm problem. Results of numerical simulation for the parameters used show that significantly faster tracking is obtained with the proposed control law, thus establishing the utility of the method. However, more work is required to develop better estimates of the time to OTE.

\section{References}

Bartoszewicz A 1995 A comment on a time varying sliding surface for fast and robust tracking control of second-order uncertain systems. Automatica 31: 1893-1895

Bartoszewicz A 1996 Time-varying sliding modes for second-order systems. IEE Proc. Control Theor. Appl. 143: 455-462

Chiacchiarini H G, Desages A C, Romagnoli J A, Palazoglu A 1995 Variable structure control with a second-order sliding condition: Application to a steam generator. Automatica 31: 1157-1168

Choi S, Park D, Jayasuriya S 1994 A time varying sliding surface for fast and robust tracking control of second-order uncertain systems. Automatica 30: 899-904

DeCarlo R A, Zak S H, Matthews G P 1988 Variable structure control of nonlinear multivariable systems: A tutorial. Proc. IEEE 76: 212-232

Drazenovic B 1969 The invariance conditions in variable structure systems. Automatica 5: 287-295 
Gao W, Hung J C 1993 Variable structure control of nonlinear systems: A new approach. IEEE Trans. Ind. Electron. 40: 45-55

Hung J Y, Gao W, Hung J C 1993 Variable structure control: A survey. IEEE Trans. Ind. Electron. 40: $2-21$

Mantz R J, Battista H, Puleston P 2001 A new approach to reaching mode of VSS using trajectory planning. Automatica 37: 763-767

Singh G K 1999 Tracking error based sliding mode controller design with application to fight control. $\mathrm{Ph} \mathrm{D}$ thesis, Indian Institute of Technology, Kanpur

Singh S N 1989 Asymptotically decoupled discontinuous control of systems and nonlinear aircraft maneuver. IEEE Trans. Aerosp. Electron. Syst. 25: 380-391

Slotine J J E, Li W 1991 Applied nonlinear control (Englewood Cliffs, NJ: Prentice Hall)

Utkin V I 1977 Variable structure systems with sliding mode. IEEE Trans. Autom. Control 22: 212-222

Utkin V I 1978 Sliding modes and their applications in variable structure systems. (Moscow: Mir)

Wheeler G, Chun-Yi Su, Yury S 1998 A sliding mode controller with improved adaptation laws for the upper bounds on the norm of uncertainties. Automatica 34: 1657-1661

Yilmaz C, Hurmuzlu Y 2000 Eliminating the reaching phase from variable structure control. Trans. ASME: J. Dyn. Syst. Meas. Control 122: 753-757

Young K D, Utkin V I, Oezguener U 1999 A Control engineer's guide to sliding mode control. IEEE Trans. Control Syst. Technol. 7: 328-342 